University of Wollongong

Research Online

Faculty of Engineering and Information

Faculty of Engineering and Information

Sciences - Papers: Part A

Sciences

$1-1-2020$

Hybrid Translation and Language Model for Micro Learning Material Recommendation

Jiayin Lin

University of Wollongong, j1461@uowmail.edu.au

Follow this and additional works at: https://ro.uow.edu.au/eispapers

Part of the Engineering Commons, and the Science and Technology Studies Commons

Research Online is the open access institutional repository for the University of Wollongong. For further information contact the UOW Library: research-pubs@uow.edu.au 


\title{
Hybrid Translation and Language Model for Micro Learning Material Recommendation
}

\begin{abstract}
As an emerging pedagogy, micro learning aims to make use of people's fragmented spare time and provide personalized online learning service, for example, by pushing fragmented knowledge to specific learners. In the context of big data, the recommender system is the key factor for realizing the online personalization service, which significantly determines what information will be fmally accessed by the target learners. In the education discipline, due to the pedagogical requirements and the domain characteristics, ranking recommended learning materials is essential for maintaining the outcome of the massive learning scenario. However, many widely used recommendation strategies in other domains showed defectiveness in the ability to rank the recommended results. In this paper, we propose a novel recommendation strategy based on the combination of the language model and the translation model. The proposed recommendation strategy aims to filter out unsuitable learning materials and ranks the recommended learning materials more effectively.
\end{abstract}

\section{Keywords}

recommendation, material, translation, learning, language, hybrid, micro, model

\section{Disciplines}

Engineering | Science and Technology Studies

\section{Publication Details}

Lin, J. (2020). Hybrid Translation and Language Model for Micro Learning Material Recommendation. IEEE International Conference on Advanced Learning Technologies (pp. 384-386). United States: IEEE. 


\title{
Hybrid Translation and Language Model for Micro Learning Material Recommendation
}

\author{
Jiayin Lin \\ School of Computing and Information Technology, \\ University of Wollongong, Wollongong, NSW, Australia \\ j1461@uowmail.edu.au
}

\begin{abstract}
As an emerging pedagogy, micro learning aims to make use of people's fragmented spare time and provide personalized online learning service, for example, by pushing fragmented knowledge to specific learners. In the context of big data, the recommender system is the key factor for realizing the online personalization service, which significantly determines what information will be finally accessed by the target learners. In the education discipline, due to the pedagogical requirements and the domain characteristics, ranking recommended learning materials is essential for maintaining the outcome of the massive learning scenario. However, many widely used recommendation strategies in other domains showed defectiveness in the ability to rank the recommended results. In this paper, we propose a novel recommendation strategy based on the combination of the language model and the translation model. The proposed recommendation strategy aims to filter out unsuitable learning materials and ranks the recommended learning materials more effectively.
\end{abstract}

Keywords- Recommender System, Information Filtering, Micro Learning, Big Data, Machine Learning

\section{INTRODUCTION}

The soaring development of the Internet and mobile devices gave birth to an emerging type of online learning service micro learning [1], which has been aiming to utilise users' fragmented spare time and carry out portable learning activities. As the key to the personalized online learning service, the recommendation strategy determines what information will be finally delivered to the target user. Manually managing massive online learning resources and user's information is significantly labour-intensive and costly, and the workload is beyond imagination. Hence, a delicate recommender system has the potential to significantly improve the learning outcomes and reduce the dropout rate for formal, informal or non-formal online learning service.

In the context of big data, a solid recommender system is essential for guaranteeing the efficiency and accuracy of online service. Nowadays, the boundary between information filtering (i.e. recommender system) and information retrieval (i.e. the main function of search engine) becomes blurred. Both of them have a similar objective: finding and delivering relevant information to the target users [2]. In order to satisfy users' personalized online learning requirements and overcome the difficulty in finding suitable information from a large repository of knowledge, a recommender system should also take the items ranking into account. Also the study [3] argues that there is little research about applying classic information retrieval (IR) techniques to boost the performance of a recommender system (RS). It is argued that the probabilistic model has a solid statistical foundation hence has the potential to make significant improvements to the field of recommender system as it did in IR [2].

When a recommender system formulates a list of recommended learning activities to a target user, it is actually distilling a user's online historical learning activities and the relevant information from the digital learning materials into a list of recommendations.

In this paper, we analyse current gaps in realizing micro learning service and propose a hybrid translation and language model for ranking and recommending personalized online learning materials to online learners using mobile devices. The remainder of the paper is organised as follows. Section II will bring out the problems and gaps in this area. Relevant research questions will also be proposed in this section. The proposed solution, methodology, and evaluation metrics will be discussed in Section III. The expected contribution and plans for the future work will be introduced in Section IV.

\section{RELATED WORK}

In one previous work on information retrieval [4], researchers developed their information retrieval model based on the ideas and methods of statistical machine translation. Moreover, they proved that deeming documents retrieval as a problem in translation might not be a fanciful idea but still a feasible one. In [4], a probabilistic approach was used to estimate the mapping probability between a certain query and document pair. Such mapping process was modelled based on the statistical model proposed by the authors. In another work with regard to boosting the performance of information retrieval [5], the authors used a language model to denote the relationship between the documents and queries. In a nutshell, authors approximated the relevant degree between a query and a document by the probability of co-occurrence between a word $\mathrm{w}$ from the document and the query.

In the IR research field, statistical language models were explored to handle the recommendation task [3]. Different smoothing and prior probability estimation methods were compared in the experiments, which also indicated that the probabilistic modelling method for the recommender system did have the potential to capture some latent information missed by matrix factorization. However, in the study [3], the recommending process is not treated as a mapping procedure between two different sources. 


\section{Problem Statement And Research Questions}

\section{A. The Defects of Algebraic-based Recommendation Models}

Even though algebraic-based recommender system has been demonstrated to be very effective in predicting items' rating value, it lacks the ability to precisely represent the ranking of recommended items. Moreover, the training process of most matrix factorization based recommender systems is guided by the rating value [6]. It does not involve any explicit features, which could not represent the ranking information among items. As discovered in one study [3], algebraic-based collaborative filtering cannot generally provide good result in the top-k recommendation task. Hence, the authors of this study argue that the probabilistic method can be a more effective and formal way for generating personalized rankings of recommendations [3].

\section{B. The Demand of Ranking Model for Pedagogical Requirement}

As a novice online learning service, a micro learning system consists of three significant intelligent modules, i.e., non-micro learning material segmentation, micro learning material annotation, and micro learning material recommendation. The workflow of this online learning service is proposed in one previous study [7]. For personalized online service, both segmentation and annotation modules focus on managing online materials, whereas the recommendation module directly interacts with users for information exchange during the online learning activity. As these three intelligent modules are highly cohesive and loosely coupled, in this paper, we specifically concentrate on illustrating the recommendation strategy for this system. Segmentation for non-micro learning materials and annotation for less descriptive learning materials are beyond the scope of this paper.

Significantly different from the recommender systems used in other domains of applications, such as e-commerce, in e-learning, pedagogical issues $[8,9]$ or requirements are always involved in the design and development of online service and influencing the decision-making progress. For example, the description of a learning material might always contain vague information and a learner's preference might not be pedagogically appropriate for him/her. Hence, a recommender system that could precisely rank the learning materials would greatly boost the outcome of personalised learning activities.

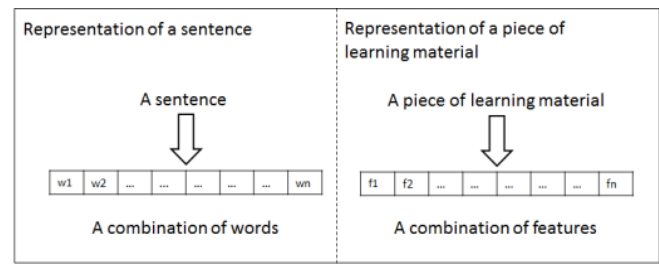

Figure 1.The similar representation of a sentence and a piece of learning material

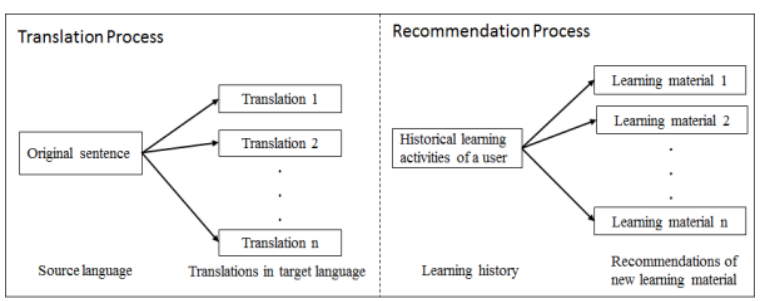

Figure 2.The similar process of translation and recommendation

\section{Research Questions}

Based on the resource we currently have, the reviewed literature, and my personal research background, the research question can be condensed as follow:

1. How to design a delicate recommender system for the micro learning service?

As discussed above, micro learning service requires a recommending strategy which can rank the candidate of the learning resource. Moreover, due to several limitations, conventional algebraic-based recommendation strategies are not suitable for the micro learning service. The probabilistic method might be a more effective and formal way of generating personalised ranks of recommendations.

2. What features/aspects should be involved when designing a solid recommendation strategy?

For personalised recommendation of micro learning resource: the recommendation strategy should be based on analysing user's historical learning records, finding similar learners and mining user's learning requirement and knowledge level.

\section{PROPOSED METHODOLOGY}

\section{A. Hybrid Translation and Language Model}

The statistical language model [10], reflects the combinational relationship of the features or metadata, which represents a certain object, while the statistical machine translation model reflects the mapping relationship between two different objects, such as the Bayes Theorem used in [11]. A representation procedure for a learning material can be treated as a language modelling problem.

Comparison of language modelling and learning material modelling is shown in Figure 1. A sentence is represented by a series of words distributed in a sequential manner; a piece of learning material is represented by features. If the involved features of a learning material have sequential relationships, it can be represented in a similar way. For a recommendation task in e-learning or micro learning as tackled in this paper, to recommend new learning materials to the target users based on his/her historical learning activities, can be seen as a translation modelling problem, a mapping procedure between historical learning activities and new learning materials. These two similar processes are shown in Figure 2. During the process of statistical machine translation, a sentence from one language could have different probabilities to be translated to different results in another language; and the higher 
probability, the more reasonable the translation result. Based on this formal concept, the translation procedure can be modelled in a similar way: for a certain learning history of a user, there could be several different recommended materials with different probabilities, the higher probability means this recommended material is more suitable for this user.

Hence, in this paper, we propose a novel recommendation strategy based on the combination of the language model and translation model. A translation model is used to model the mapping process of historical learning records and the recommended learning materials; a language model is used to model the representation of certain learning material, our recommendation strategy can be formulated as:

$$
P(l \mid h) \propto \prod_{i=1}^{k} P\left(f_{i} \mid f_{i-(n-1)}, \ldots, f_{i-1}\right) P(h \mid l)
$$

Herein, $l$ represents the learning material, $h$ represents that historical learning records of the target user, $f_{i}$ is the $i$-th feature that is used to represent the leaning material. The probability $\mathrm{P}(\mathrm{l} \mid \mathrm{h})$ represents the degree of correlation between a user's historical learning activities and the new learning material. Finding the best recommendation result $\hat{l}$ is realised by finding the one that gives the highest probability.

$$
\hat{\mathrm{l}}=\underset{\mathrm{e}}{\operatorname{argmax}} \prod_{\mathrm{i}=1}^{\mathrm{k}} \mathrm{P}\left(\mathrm{f}_{\mathrm{i}} \mid \mathrm{f}_{\mathrm{i}-(\mathrm{n}-1)}, \ldots, \mathrm{f}_{\mathrm{i}-1}\right) \mathrm{P}(\mathrm{h} \mid \mathrm{l})
$$

\section{B. Evaluation Metrics}

\section{1) Normalized Discounted Cumulative Gain (NDCG)}

The proposed recommendation strategy aims to alleviate the defective ranking ability of conventional recommendation models for micro learning system. Hence, precisely measuring and comparing the ranking ability of the models is significant. Normalized Discounted Cumulative Gain (NDCG) [12] could avoid the influence of comparing ranking ability between recommendation lists with different lengths.

2) Precision and Recall at Top $K$

In most cases, for a recommended item list, a user only views only the top $\mathrm{k}$ results. Hence, it is more reasonable that we only consider top $\mathrm{k}$ recommended results in the experiments. Precision is the fraction of relevant items in the list of recommendations; precision@ $\mathrm{k}$ reflects the fraction of relevant items when only considering top $\mathrm{k}$ items in the recommendation list. The recall is the fraction of relevant items retrieved over all relevant items; recall@ $\mathrm{k}$ is the number of relevant items found in the top $\mathrm{k}$ recommendations.

\section{EXPECTED CONTRIBUTION AND FUTURE WORK}

In this paper, we propose a hybrid translation and language model for ranking and recommending personalized online learning materials to online learners using mobile devices. The expected contribution of this work will be a novel recommender system which can precisely recommend the target user with personalised learning materials. Such recommender system may be made up of multiple intelligent analysis and summarising parts, and should have the ability mining, distilling, and summarising different the type of information involved in the learning activity.
In the near future, we will continue carrying out the experiments of the proposed recommendation strategy. The recommendation strategy proposed in this study is an initial prototype of our ongoing research on the recommending strategy for micro-learning materials. Later, we will focus our work in the area: Optimizing the representation of the language model and translation model. There are several different ways to model the mapping relationship and the representation of online learning materials. Neural networks and embedding techniques have been proved to be effective in constructing a language model [13] and a translation model [14].

\section{ACKNOWLEDGEMENT}

This research has been carried out with the support of the Australian Research Council Discovery Project, DP180101051, and Natural Science Foundation of China, no. 61877051, and UGPN RCF 2018-2019 project between University of Wollongong and University of Surrey.

\section{REFERENC}

[1] G. Sun, T. Cui, J. Yong, J. Shen and S. Chen, MLaaS: a cloud-based system for delivering adaptive micro learning in mobile MOOC learning. IEEE Transactions on Services Computing, 2018. 11(2): p. 292-305

[2] N.J. Belkin and W.B. Croft. Information filtering and information retrieval: two sides of the same coin. in Communications of the ACM. 1992. Citeseer.

[3] D. Valcarce. Exploring statistical language models for recommender systems. in Proceedings of the 9th ACM Conference on Recommender Systems. 2015, 375-378. ACM.

[4] A. Berger and J. Lafferty. Information retrieval as statistical translation. in ACM SIGIR Forum. 2017, 219-226. ACM.

[5] V. Lavrenko and W.B. Croft. Relevance-based language models. in ACM SIGIR Forum. 2017, 260-267. ACM.

[6] Y. Koren, R. Bell and C. Volinsky, Matrix factorization techniques for recommender systems. Computer, 2009(8): p. 30-37.

[7] J. Lin, G. Sun, J. Shen, T. Cui, P. Yu, D. Xu and L. Li. A Survey of Segmentation, Annotation, and Recommendation Techniques in Micro Learning for Next Generation of OER. in 2019 IEEE 23rd International Conference on Computer Supported Cooperative Work in Design (CSCWD). 2019, 152-157. IEEE.

[8] D. Wu, J. Lu and G. Zhang, A fuzzy tree matching-based personalized e-learning recommender system. IEEE Transactions on Fuzzy Systems, 2015. 23(6): p. 2412-2426.

[9] R. Sikka, A. Dhankhar and C. Rana, A survey paper on e-learning recommender system. International Journal of Computer Applications, 2012. 47(9): p. 27-30.

[10] P.F. Brown, P.V. Desouza, R.L. Mercer, V.J.D. Pietra and J.C. Lai, Class-based n-gram models of natural language. Computational linguistics, 1992. 18(4): p. 467-479.

[11] P.F. Brown, V.J.D. Pietra, S.A.D. Pietra and R.L. Mercer, The mathematics of statistical machine translation: Parameter estimation. Computational linguistics, 1993. 19(2): p. 263-311.

[12] Y. Wang, L. Wang, Y. Li, D. He, W. Chen and T.-Y. Liu. A theoretical analysis of NDCG ranking measures. in Proceedings of the 26th annual conference on learning theory (COLT 2013). 2013, 6.

[13] Y. Kim, Y. Jernite, D. Sontag and A.M. Rush. Character-aware neural language models. in Thirtieth AAAI Conference on Artificial Intelligence. 2016.

[14] R. Sennrich, B. Haddow and A. Birch, Neural machine translation of rare words with subword units. arXiv preprint arXiv:1508.07909, 2015. 